\title{
Retrospective Review of Emergency Response Activations During a 13-Year Period at a Tertiary Care Children's Hospital
}

\author{
George Sam Wang, MD \\ Nicole Erwin, BA $^{2}$ \\ Jeannie Zuk, PhD, RN ${ }^{3}$ \\ Desmond B. Henry, мо ${ }^{4}$ \\ Emily L. Dobyns, MD $^{5}$
}

\author{
${ }^{1}$ Section of Emergency Medicine, The Children's Hospital, Aurora, Colorado. \\ ${ }^{2}$ University of Colorado Health and Sciences, Aurora, Colorado. \\ ${ }^{3}$ Department of Anesthesia, The Children's Hospital, Aurora, Colorado. \\ ${ }^{4}$ Department of Anesthesia, University of Texas Southwestern, Dallas, Texas. \\ ${ }^{5}$ Section of Critical Care Medicine, The Children's Hospital, Aurora, Colorado.
}

Disclosure: Nothing to report.

OBJECTIVES: Pediatric in-hospital arrests are uncommon but are associated with poor outcomes. In preparation for implenting a Rapid Response Team (RRT) at The Children's Hospital, we reviewed our data collection of 13 years of emergency response team (ERT) activations. We describe demographic and clinical variables, including outcomes of ERT activations at a free-standing tertiary care children's hospital.

METHODS: Analysis was performed on data collected from January 1993 through July 2007. Variables collected included age, sex, admission diagnosis, core event, admission diagnosis and secondary diagnosis, medical division or winter/nonwinter months, day/night shifts, survival of core event, survival to discharge, and primary attending service.

RESULTS: There were 1537 ERT activations in the database, 203 were eliminated due to missing data or were adult visitors/ employees. The remaining 1334 were included for analysis. Our results showed 39\%(511) of all ERT activations occurred in patients under 1 year of age. The most common admission diagnosis category was cardiac disease. There was no statistical significance between summer and winter months although more activations occurred during daytime hours $(P<.001)$. Survival rate of an ERT was $90 \%$, with a $78 \%$ survival rate to discharge.

CONCLUSION: Our data support the general belief that younger children with chronic disease are at highest risk for ERT activations. These risk factors should be taken into consideration when planning patient placement, medical staffing, and the threshold for ICU consultations or admissions. More extensive multisite studies using clinical data are necessary to further identify hospitalized children at risk for sudden decompensation. Journal of Hospital Medicine 2011;6:131-135. @ 2010 Society of Hospital Medicine.

KEYWORDS: cardiac or respiratory event, emergency response team, pediatrics, rapid response team, the Children's Hospital.

The incidence of sudden pediatric cardiac or respiratory arrest is low. ${ }^{1}$ Most inpatient pediatric arrests appear to occur as progression of respiratory distress or shock. ${ }^{2}$ The outcome of inpatient pediatric cardiorespiratory arrests continues to be poor, emphasizing the need for early recognition and intervention. In 2006, as part of its "100,000 Lives Campaign" the Institute for Healthcare Improvement recommended the implementation of Rapid Response Teams (RRT) as 1 of the strategies to reduce the number of preventable inpatient deaths. ${ }^{3}$ We reviewed all emergency response team (ERT) activations for last 13 years at The Children's Hospital in Denver, CO to assist in the development of a new RRT and to identify at risk populations, situations, and system processes the RRT should address.

This is a retrospective review of 13 years of data collection on ERT activations at The Children's Hospital in Denver, CO. We describe demographic and clinical variables, including outcomes of ERT activations at a free-standing tertiary care children's hospital.

\section{Background/Methods}

The Children's Hospital (TCH) is a 270 inpatient bed tertiary care free standing children's hospital associated with University of Colorado at Denver Health Sciences Center. The current distribution of inpatient beds includes: 168 medical/ surgical beds, 102 critical care beds (26 pediatric intensive care unit, 16 cardiac intensive care unit, and 60 neonatal intensive care unit). In 2006, nearly 10,000 patients were admitted for care at this hospital with an average inpatient stay of 6 days. TCH is a Level One Regional Trauma Center serving a catchment area of seven states, and a major transplant center for heart, solid organs, and bone marrow.

The history of the TCH Emergency Response Team dates back to 1990 when we first began to follow cardiorespiratory arrests in noncritical care areas of the hospital. In 1992, a Cardiac Or Respiratory event (COR) team including the most senior in-house specialist was available 24 hours a day, 7 days a week to respond to all arrests within the hospital. The COR committee provided oversight and monitoring of the arrest events, including standardization of crash carts 


\begin{tabular}{|c|c|}
\hline Variable $(n=1334)$ & n (\%) \\
\hline \multicolumn{2}{|l|}{ Age } \\
\hline Neonate & $127(10)$ \\
\hline$<1$ year & $384(29)$ \\
\hline $1-3$ years & $324(24)$ \\
\hline 4-6 years & $137(10)$ \\
\hline $7-10$ years & $120(9)$ \\
\hline 11-13 years & $84(6)$ \\
\hline $14-17$ years & $120(9)$ \\
\hline$>17$ years & $38(3)$ \\
\hline \multicolumn{2}{|l|}{ Gender } \\
\hline Male & $807(60)$ \\
\hline \multicolumn{2}{|l|}{ Time of day* } \\
\hline Day (06:00-18:00) & $784(59)$ \\
\hline Night (18:00-06:00) & $550(41)$ \\
\hline \multicolumn{2}{|l|}{ Time of Year } \\
\hline April-September & $669(50)$ \\
\hline October-March & $665(50)$ \\
\hline $\begin{array}{l}\text { Abbreviation: ERT, emerge } \\
{ }^{*} P<0.001 \text {. }\end{array}$ & \\
\hline
\end{tabular}

and the development of mock codes to ensure that the responding personnel were qualified in resuscitation practices. The COR team has evolved over the ensuing years, including a name change to the Emergency Response Team (ERT); now a single number activated by any medical staff is used to call the operator who activates the ERT via overhead paging and via code pager system. The 14 member Emergency Response Team consists of PICU/ $\mathrm{CICU} /$ anesthesia/surgical fellows, ED attending, in-house residents, PICU/CICU/ED charge nurse, nursing supervisor, resource $\mathrm{RN}$, pharmacist, respiratory therapist, and a messenger.

A database has been maintained by 1 of the authors $(\mathrm{DBH})$ since the inception of the COR/ERT at TCH 16 years ago. This is a retrospective review of this database of ERT activations. An ERT activation could have been triggered by any event that was felt to be emergent, life threatening, and/or needing immediate medical attention. After the event, a debriefing form was filled out about the event. Data collected included date, time, medical record number, location, primary care service, age, sex, primary and secondary diagnoses, and disposition. Data not captured in this database included initial rhythm, need for compressions, cardiac medications, defibrillation or intubation.

Analyses were performed on data collected from January 1993 through April 2007. Medical records of the documented ERT activations were reviewed for missing information and/or clarification of the events. Categories entered in Statistical Package for Social Statistics (SPSS) were similar to information included in the debriefing form: age, sex, admission diagnosis, precipitating event, percentage of
TABLE 2. Admission Diagnosis Categories of ERT

\section{Activation Patients}

\begin{tabular}{lc} 
Admission Diagnosis $(\mathbf{n}=\mathbf{1 3 3 4})$ & $\mathbf{n}(\mathbf{\%})$ \\
\hline Cardiac & $370(28)$ \\
Infectious disease & $197(15)$ \\
Trauma & $192(14)$ \\
Other medical & $137(10)$ \\
Pulmonary & $109(8)$ \\
Neurology & $79(6)$ \\
Other surgery & $76(6)$ \\
Neurosurgery & $68(5)$ \\
Hematology/oncology & $53(4)$ \\
Surgery & $53(4)$ \\
\hline & \\
\hline
\end{tabular}

admissions, acute vs. chronic diagnosis, winter vs. nonwinter months (October-March/April-September), day (6 am-6 pm) and night (6 pm-6 am) shifts, survival of ERT activation, survival to discharge, and primary attending service. Data were analyzed using SPSS 16.0 (2007, Chicago, IL). The study was approved by the Colorado Multiple Institutional Review Board.

\section{Results/Conclusion}

There were 1537 ERT activations in the database. A total of 203 ERT activations were eliminated from the database: 177 were eliminated from analysis because of missing age, admission diagnosis or time of day of activation, and 26 were ERT activations that had been triggered on adult visitors or adult employees. The remaining 1334 ERT activations were included for analysis.

Table 1 shows the demographics of the patients. The median age was 1.8 years, with a range of 0 to 29 years. A total of $39 \%(511)$ of all ERT activations occurred in patients under the age of 1 year with the highest incidence between 1 month and 1 year. Overall, the children at highest risk were males less than 1 year of age with a chronic diagnosis. In addition, time of day and time of year of ERT activations were analyzed as shown. There was no statistical difference between nonwinter (April-September) and winter (OctoberMarch) months. Statistically, there were significantly more ERT activations during day shifts (6 am-6 pm) as compared to night shifts $(P<0.001)$.

The most common admission diagnosis (Table 2) and underlying chronic condition was cardiac disease; other common admission diagnoses were infectious disease, trauma, and pulmonary disease. The medical categories of admission diagnosis included congenital/metabolic (39\%), gastrointestinal $(29 \%)$, renal $(18 \%)$, rheumatology $(4 \%)$, toxicology $(4 \%)$, psychiatry $(3 \%)$, endocrine $(2 \%)$, and allergy (1\%). The surgery category of admission diagnosis included otolaryngology $(63 \%)$, orthopedics $(28 \%)$, urology $(4 \%)$, dental $(4 \%)$, and ophthalmology (1\%). 


\section{TABLE 3. Survival of Event of ERT Activation Patients}

\begin{tabular}{lc} 
Admission Diagnosis & Survival of Event $\mathbf{n}(\%)$ \\
\hline Cardiac $(\mathrm{n}=370)$ & $315(85)$ \\
Infectious disease $(\mathrm{n}=197)$ & $186(94)$ \\
Trauma $(\mathrm{n}=191)$ & $168(88)$ \\
Other medical $(\mathrm{n}=136)$ & $125(92)$ \\
Pulmonary $(\mathrm{n}=109)$ & $97(89)$ \\
Neurology $(\mathrm{n}=79)$ & $75(95)$ \\
Other surgery $(\mathrm{n}=76)$ & $75(99)$ \\
Neurosurgery $(\mathrm{n}=68)$ & $63(93)$ \\
Surgery $(\mathrm{n}=53)$ & $50(94)$ \\
Hematology/oncology $(\mathrm{n}=53)$ & $46(87)$ \\
Total $(\mathrm{n}=1332)^{*}$ & $1200(90)$ \\
\hline
\end{tabular}

Abbreviation: ERT, emergency response team.

*Two patients missing survival event data.

Finally, the patients' survival rate after an ERT itself was to be $90 \%$ (Table 3), with an overall survival rate to discharge of $78 \%$ (Table 4). Survival rate to discharge of those patients who survived the ERT event was $87 \%$. Two patients were missing survival event data and 137 patients were missing survival to discharge data.

\section{Discussion}

We present a retrospective review of 1334 emergency response team activations over 13 years at an academic free-standing tertiary care children's hospital. In keeping with previous reports, we found that children less than 1 year of age were at the highest risk for activation of the emergency response team. ${ }^{1,4,5}$

The National Registry for CardioPulmonary Resuscitation (NRCPR) database cite respiratory failure (asphyxia) and circulatory shock (ischemia) as the most common causes of in-hospital cardiac arrests. ${ }^{1}$ Additionally, more than half of pediatric patients that experience a cardiopulmonary arrest have an underlying chronic illness. ${ }^{1,2,4,5,6}$ These are similar to our findings that chronic pulmonary and cardiac diseases were among the most frequent admission diagnosis.

Unlike Peberdy et al., ${ }^{7}$ we did not find an increase in the number of emergency response team activations at night or on weekends. Instead, we found that an ERT activation was more likely to be requested during the day shifts (6 am-6 $\mathrm{pm}$ ) which is a similar to that reported by Jones et al. ${ }^{8}$ Similarly, Jones et al. ${ }^{8}$ reported that the hourly rate of their medical emergency team activation was greater during the time between 8 am and $6 \mathrm{pm}$.

Our overall survival rate (78\%) to discharge after an ERT event was much higher than what has been reported by Topjian et al. ${ }^{9}$ (25\%). This likely reflects our inclusion of all emergency response team activations, not just apneic and asystolic arrests. The improved survival rate may also be influenced by the $24 / 7$ presence of pediatric fellows, residents and surgeons in our hospital, which has been associ-

\section{TABLE 4. Survival to Discharge of ERT Activation}

\section{Patients}

\begin{tabular}{lc} 
Admission Diagnosis & Survival to Discharge $\mathbf{n}(\%)$ \\
\hline Cardiac $(\mathrm{n}=317)$ & $217(69)$ \\
Infectious disease $(\mathrm{n}=175)$ & $139(79)$ \\
Trauma $(\mathrm{n}=182)$ & $151(83)$ \\
Other medical $(\mathrm{n}=125)$ & $99(79)$ \\
Pulmonary $(\mathrm{n}=98)$ & $76(78)$ \\
Neurology $(\mathrm{n}=71)$ & $63(89)$ \\
Other surgery $(\mathrm{n}=74)$ & $70(95)$ \\
Neurosurgery $(\mathrm{n}=64)$ & $53(83)$ \\
Surgery $(\mathrm{n}=47)$ & $39(83)$ \\
Hematology/oncology $(\mathrm{n}=44)$ & $24(55)$ \\
Total $(\mathrm{n}=1197)^{*}$ & $931(78)$ \\
\hline
\end{tabular}

Abbreviation: ERT, emergency response team.

${ }^{*}$ Total of 137 patients missing survival discharge data.

ated with improved 24-hour survival for children receiving in-hospital cardiopulmonary resuscitation. ${ }^{10}$

There are several limitations to our data collection and this report. This was a retrospective review and as previously noted some medical details were absent resulting in the exclusion of some cases. We also found that the original debriefing form which was used as the basis for the database did not include some important clinical variable, such as vital signs, more detailed events such as rhythm, medications used, and deficits or changes in baseline function. We suggest including multiple variables in a future multicenter study of pediatric RRT's: facility, admitting service, admission diagnosis, age (chronologic and gestational), sex, how long patient has been in hospital prior to the event, past medical history, vitals signs before and after activation, time/date, location, any precipitating events and actions taken by the RRT (eg, cardiopulmonary resuscitation, defibrillation, emergent intubation, and other emergent interventions), medications, survival of event, survival to discharge, deficits or changes in baseline function after the event and to discharge. In particular, information about history of prematurity would have been helpful in assessing further risk factors. Analysis of survival with or without significant deficits or changes in baseline function would be another useful outcome measure. Figure 1 is an example of a debriefing form a multi-institutional study or hospitalist led quality improvement project may use to collect this data.

We were able to identify a population of higher-risk patients (less than 1 year of age with comorbidities and an admission diagnosis of cardiac or respiratory disease) to focus our educational efforts on earlier recognition of patient deterioration for both inpatient ward staff and RRT responders. These findings may assist in future quality assurance issues such as patient placement and early ICU admissions depending on age, chronic conditions, and/or 
GENERAL INFORMATION:

1. Date and time of resuscitation:

2. Admitting Service/Attending MD:

3. Names of personnel involved in event, level of training:

4. Primary/admit diagnosis:

5. Past Medical history:

6. Event precipitating arrest:

7. Who initiated Code Blue?

8. $\quad$ Text message on Code Blue pagers clear and called at appropriate time? (if no explain)

9. Elapse time for arrival:

INITIAL ASSESSMENT:

10. RN/ staff assessment indicated that this patient might be at increased risk for requiring resuscitation. List factors:

$\begin{array}{lllll}\text { 11. Vital signs prior to activation: HR: } & \text { BP: } & \text { RR: } & \text { Pulse Ox: } & \text { Temp: }\end{array}$

IMPLEMENTATION:

\begin{tabular}{|c|l|l|l|}
\hline 12. & Airway, breathing and circulation were established prior to the arrival of the Code Team. & & \\
\hline 13. & Equipment (cor cart, defibrillator, etc.) was readily available and in working order. If no, list below: & & \\
\hline 14. & Appropriate drugs available? & & \\
\hline 15 & Appropriate personnel responded? If no, explain: & & \\
\hline 18. & Communications among disciplines/personnel were clear? If no, explain: & & \\
\hline 19. & Family Present during resuscitation? & & \\
\hline
\end{tabular}

EVALUATION:

20. Elapse time of resuscitation:

21. Disposition of patient:

22. Event predictable and/or preventable? Explain:

EFFECTIVENESS OF INTERVENTIONS (Describe if problems)

\begin{tabular}{|l|l|l|l|}
\hline 23 & Vital signs after resuscitation: HR: BP: Pulse Ox: & & \\
\hline 24. & Bag and Mask/Time & & \\
\hline 25. & Chest Compressions/Time & & \\
\hline 26 & IV/IO access/Time to access: & & \\
\hline 27 & Defibrillation/Cardioversion & & \\
\hline 28. & Resuscitation Medications/dosing and amount: & \\
\hline 29. & $\begin{array}{l}\text { Intubation Method (circle): Awake Rapid Sequence IV none By whom: } \\
\text { Attempts: }\end{array}$ & & \\
\hline 31 & Survival of Event? To discharge? & & \\
\hline 32 & Change in baseline function i.e. POPC score? At discharge? & \\
\hline
\end{tabular}

35. Please list aspects of the resuscitation that you feel were performed well:

36. Please list aspects of the resuscitation that could have been performed better:

Name \& Phone Number of Person Completing Form:

FIGURE 1. Code Blue Activation Follow-Up and Debriefing Form.

admission diagnosis. Future directions should include multi-center study of RRT to improve external validity. In addition, a more careful analysis of events surrounding the activation, including incorporating such tools as the Pediatric Early Warning (PEW) Score, ${ }^{11}$ may further assist hospitals and practitioners identify hospitalized children at risk for deterioration on the inpatient ward.

\section{Acknowledgements}

The authors acknowledge Dr. Genie Roosevelt MD, MPH, and Sara Deakyne MPH for their assistance in data analysis.

Address for correspondence and reprint requests:

George Sam Wang, MD, 13123 E 16th Ave B251, Aurora, CO 80045; E-mail: Wang.sam@tchden.org Received 12 March 2009; revision received 3 March 2010; accepted 2 July 2010. 


\section{References}

1. Berg MD, Nadkarni VM, Zuercher M, Berg RA. In-hospital pediatric arrest. Pediatr Clin North Am. 2008;55(3):589-604.

2. Nadkarni VM, Larkin GL, Peberdy MA, et al. First documented rhythm and clinical outcome from in-hospital cardiac arrest among children and adults. JAMA. 2006;295:50-57.

3. $57100 \mathrm{~K}$ Lives Campaign-getting started Kit: Rapid response Teams. Available at: http://www.ihi.org/IHI/Programs/Campaign/Campaign.htm? TabId=1 Accessed July 2010.

4. Meaney PA, Nadkarni VM, Cook EF, et al. Higher survival rates among younger patients after pediatric intensive care unity cardiac arrests. Pediatrics 2006;118:2424-2433.

5. Reis A.G, Nadkarni V.M. et al. A prospective investigation into the epidemiology of in-hospital pediatric cardiopulmonary resuscitation using the international Utsein Reporting Style. Pediatrics. 2002;109: 200-209.
6. Gillis J, Dickson D, Rieder M, Steward D, Edmonds J. Results of inpatient pediatric resuscitation. Crit Care Med. 1986;14:469-471

7. Peberdy MA, Ornato JP, Larkin GL, et al. Survival from in-hospital cardiac arrest during nights and weekends. JAMA. 2008;299:785-792.

8. Jones D, Bates S, Warrillow S, et al. Circadian pattern of activation of the medical emergency team in a teaching hospital. Crit Care. 2005;9:R303-R306.

9. Topjian AA, Berg RA, Nadkarni VM. Pediatric cardiopulmonary resuscitation: advances in science, techniques, and outcomes. Pediatrics. 2008; 122;1086-1098

10. Donoghue AJ, Nadkarni VM, Elliott M, et al. Effect of hospital characteristics on outcomes from pediatric cardiopulmonary resuscitation: a report from the National Registry of Cardiopulmonary Resuscitation. Pediatrics. 2006;118:995-1001.

11. Duncan H, Hutchinson J, Parshuram CS. The Pediatric Early Warning System score: a severity of illness score to predict urgent medical need in hospitalized children. J Crit Care. 2006;21(3):271-278. 